\title{
Erratum to: Identifying candidate positive selection genes in Korean imported pig breeds
}

\author{
Wonseok Lee ${ }^{1} \cdot$ Mengistie Taye $^{1,2} \cdot$ Taehyung Kwon $^{1} \cdot$ Joon Yoon $^{3} \cdot$ Dongsung Jang ${ }^{4}$. \\ Shunsuke Suzuki ${ }^{5,6} \cdot$ Heebal Kim ${ }^{1,3,7}$
}

Published online: 4 April 2017

(C) The Genetics Society of Korea and Springer-Science and Media 2017

\section{Erratum to: Genes Genom \\ DOI 10.1007/s13258-017-0529-4}

Unfortunately, there is an error in the Acknowledgements section in the originally published article (DOI 10.1007/ s13258-017-0529-4). The correct statements are shown as follows:
Acknowledgements This work was supported by a grant from the Next-Generation BioGreen 21 Program (Project No. PJ01111501), Rural Development Administration, Republic of Korea.

The online version of the original article can be found under doi:10.1007/s13258-017-0529-4.

Heebal Kim

heebal@snu.ac.kr

1 Department of Agricultural Biotechnology, Animal Biotechnology and Research Institute of Agriculture and Life Sciences, Seoul National University, Seoul 151-921, Republic of Korea

2 Bahir Dar University, College of Agriculture and Environmental Sciences, P O Box 79, Bahir Dar, Ethiopia

3 Department of Natural Science, Interdisciplinary Program in Bioinformatics, Seoul National University, Seoul 151-921, Republic of Korea
Interdisciplinary Program in Agricultural Genomics, Seoul National University, Seoul 151-921, Republic of Korea

5 Epigenomics Division, Frontier Agriscience and Technology Center, Faculty of Agriculture, Shinshu University, Kami-Ina, Nagano, Japan

6 Department of Interdisciplinary Genome Sciences and Cell Metabolism, Institute for Biomedical Sciences, Interdisciplinary Cluster for Cutting Edge Research, Shinshu University, Kami-Ina, Nagano, Japan

7 Institute for Biomedical Sciences, Shinshu University, Kami-Ina, Nagano, Japan 